\title{
Decoupled Cascaded PID Control of an Aerial Manipulation System
}

Nebi Bulut ${ }^{1}$, Ali Emre Turgut ${ }^{1}$, Kutluk Bilge Arikan ${ }^{2}$

${ }^{1}$ Middle East Technical University, Department of Mechanical Engineering, Cankaya, Ankara, Turkey

${ }^{2}$ TED University, Department of Mechanical Engineering, Cankaya, Ankara, Turkey

\section{ABSTRACT}

\author{
Article History: \\ Received: 2019/08/10 \\ Accepted: $2019 / 12 / 09$ \\ Online: $2019 / 12 / 31$
}

Correspondence to: Nebi Bulut

Department of Mechanical Engineering,

Middle East Technical University, Çankaya,

Ankara, Turkey,

E-mail: bulutnebi@gmail.com,

Phone: +90 5075750592 combined system. The Lagrange-d'Alembert formulation is used to obtain the equation of
motion of the combined system. Later, decoupled controllers are developed for the generalized coordinates. Decoupled cascaded PID controllers are designed for trajectory tracking of the combined system. Proposed control algorithms are implemented in the MATLAB/ Simulink environment and tested using the highly nonlinear system model in simulation. The robustness of the controllers is checked by applying disturbance forces from different directions at the tip point of the 2-DOF robotic arm. The proposed control algorithms performed satisfactorily and showed very low absolute errors.

\section{Keywords:}

Aerial manipulation, Robotics, Quadrotor, PID control

\section{INTRODUCTION}

T $\mathrm{n}$ the last decade, great amount of research has been performed in the field of unmanned air vehicles (UAV). Among them, rotary wing UAV's i.e. quadrotors or their derivatives such as hexarotors and octorotors drew the most interest due to their simplicity and capabilities [1], [2], [3] and [4]. They have the ability to take-off and land vertically, they have the capability of high maneuverability and they are able to stay in hover position. They are mostly used for rescue, surveillance, and photography.

Nowadays, aerial manipulation attracts the attention of researchers since it brings new capabilities to quadrotors such as carrying payloads and manipulating objects. In most of the aerial manipulation related studies, a robotic arm is added under the UAV. In [8], [9] and [10]; serial manipulators with various degrees of freedom are studied. In [11], a parallel manipulator is exploited. [5], [6] and [7] are a few studies in which a cable suspended manipulator is used in the quadrotor.

There are mainly two different approaches for modeling of aerial manipulators. One approach is building the kinematic and dynamic model of the UAV separately and taking the effects of the manipulator such as mass and inertia as a disturbance input to the UAV as in [12] and [13]. In the other approach, the UAV and the manipulator are modeled as a single system as in [8], [9] and [17]. For the control system design of aerial manipulators, there are also different approaches in the literature. The first approach is the designing the control algorithms by considering the disturbance forces that are applied to the system. In [14], a compliance controller is implemented to overcome the interaction forces between the end-effector and the environment. To cope with interaction forces and uncertainties while providing precise trajectory tracking, a robust controller design is proposed in [15]. Another aspect of the controller design is whether a single controller is designed to stabilize the coupled system or decoupled controllers are implemented for the vehicle system. In [17] a computed torque method is used to control the UAV and the 2-DOF robot arm. In [8], a single controller is designed by using the coupled equations in order to generate control inputs for both controlling the UAV and the robotic arm. However, in [13], decoupled controllers are presented 
to regulate each state of the quadrotor and the robotic arm. The other aspect of the controller implementation is whether linear or nonlinear controllers are used to achieve stable system performance. In [16], the nonlinear model predictive control method is successfully studied to achieve optimized system performance. Linear controllers (PID) are designed to control the overall system via feedback linearized system equations [13].

In this paper, a UAV with a 2-DOF robotic arm mounted at its bottom is studied. Dynamics of the combined system is obtained by using Lagrange-d'Alembert formulation. Decoupled controllers are designed for controlling both the quadrotor and the arm. The performance and robustness of the controllers are shown using numerical simulations.

We improve the state-of-the-art in three different aspects. First, to the best of our knowledge, in most of the studies in the literature, the interaction of the end-effector with the environment is not modeled and most of the time the proposed controllers are tested without the interaction forces. However, in order to use aerial manipulators for tasks such as opening a door or pushing a box; the interaction forces have to be considered. In this paper, we considered interaction forces and designed the controller to be robust against these forces and tested its performance accordingly. Second, unlike a few studies that considered 1-D and small magnitude interaction force, we considered larger magnitude interaction forces in 3-D. Third, although the states of the aerial manipulator are highly coupled, our controller was able to control all states simultaneously unlike most of the studies in the literature.

The organization of this paper is as follows. In Section 2 , the kinematic and dynamic modeling of the system are introduced. In Section 3, controller design is discussed. The results of the simulations are presented in Section 4. Finally, in Section 5, discussion of the results and the conclusion of the study are made.

\section{MODELING}

\section{Kinematics}

\section{Position Analysis}

The position analysis is the key factor for the kinematic modeling. Here, the position relations of the combined system and Denavit-Hertenberg parameters of the robotic arm are determined [17]. Fig. 1 shows some of the kinematic parameters of the system. Let $\mathrm{O}_{i}, \mathrm{O}_{b}, \mathrm{O}_{1}, \mathrm{O}_{2}$ and $\mathrm{O}_{b}$ be the origin of the inertial, quadrotor's body, link-1, link2 and end-effector fixed reference frames, respectively.

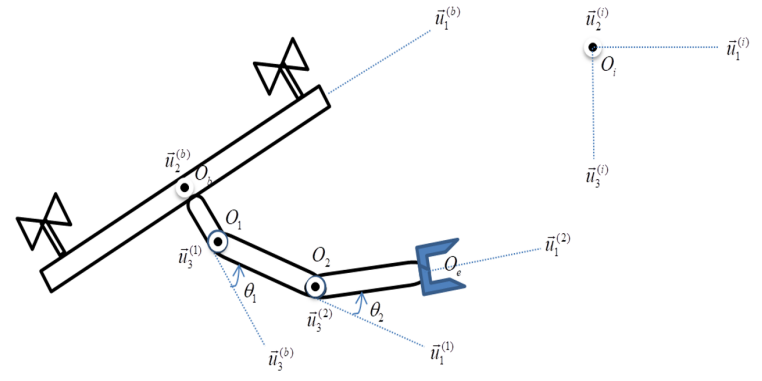

Figure 1. Side view of the unified system and some of the kinematic parameters

$\vec{u}_{k}^{(a)}$ is the $\mathrm{k}^{\text {th }}$ basis unit direction vector of the reference frame $O_{a}$. Their column matrix representation can be expressed as:

$$
\bar{u}_{1}=\left[\begin{array}{lll}
1 & 0 & 0
\end{array}\right]^{T}, \bar{u}_{2}=\left[\begin{array}{lll}
0 & 1 & 0
\end{array}\right]^{T}, \bar{u}_{3}=\left[\begin{array}{lll}
0 & 0 & 1
\end{array}\right]^{T}
$$

$\bar{\Omega}=\left[\begin{array}{ll}\theta_{1} & \theta_{2}\end{array}\right]^{T}$ is the joint angles of the robotic arm. Orientation of the center of mass of the quadrotor is represented by the set of Euler angles; roll, pitch and yaw angles $\bar{\Upsilon}=\left[\begin{array}{lll}\phi & \theta & \psi\end{array}\right]^{T}$. By using Euler angles, the rotation matrix between inertial-fixed reference frame to quadrotor bodyfixed reference frame can be written as:

$$
\hat{C}^{(i, b)}=\left[\begin{array}{ccc}
c \psi c \theta & c \psi s \theta s \phi-s \psi c \phi & c \psi s \theta c \phi+s \psi s \phi \\
s \psi c \theta & s \psi s \theta s \phi+c \psi c \phi & s \psi s \theta c \phi-c \psi s \phi \\
-s \theta & c \theta s \phi & c \theta c \phi
\end{array}\right]
$$

In Eq. (2), $s \alpha$ and $c \alpha$ denote $\sin (\alpha)$ and $\cos (\alpha)$, respectively, and $\hat{C}^{(i, b)}$ is the rotation matrix between frame $i$ to frame $b$. For the robotic arm, Denavit-Hartenberg parameters are shown in Table 1.

Table 1. Denavit-Hartenberg Parameters

\begin{tabular}{ccc}
\hline & $k=1$ & $k=2$ \\
\hline$\beta_{k}$ & $\frac{-\pi}{2}$ & 0 \\
$\theta_{k}$ & $\theta_{1}+\frac{3 \pi}{2}$ & $\theta_{2}$ \\
$b_{k}$ & $b_{1}$ & $b_{2}$ \\
\hline
\end{tabular}

Position of the quadrotor, link-1 and link-2 in the inertial-fixed reference frame can be written as:

$$
\begin{aligned}
& \bar{p}_{q}^{(i)}=\left[\begin{array}{lll}
x & y & z
\end{array}\right]^{T} \\
& \bar{p}_{1}^{(i)}=\bar{p}_{q}^{(i)}+\hat{C}^{(i, b)} \bar{p}_{1}^{(b)} \\
& \bar{p}_{2}^{(i)}=\bar{p}_{q}^{(i)}+\hat{C}^{(i, b)} \bar{p}_{2}^{(b)}
\end{aligned}
$$

$\bar{p}_{q}^{(i)}$ is the position of the quadrotor with respect to inertial frame, and $\bar{p}_{1}^{(b)}$ and $\bar{p}_{2}^{(b)}$ are the positions of the link1 and link-2 in quadrotor's body-fixed reference frame, res- 
pectively.

\section{Velocity Analysis}

The linear velocities of the quadrotor, link-1 and link-2 in the inertial-fixed reference frame can be derived by taking the time derivatives of the Eq's. (3), (4), and (5).

$$
\begin{aligned}
& \dot{\bar{p}}_{q}^{(i)}=\left[\begin{array}{lll}
\dot{x} & \dot{y} & \dot{z}
\end{array}\right]^{T} \\
& \dot{\bar{p}}_{1}^{(i)}=\dot{\bar{p}}_{q}^{(i)}+\dot{\hat{C}}^{(i, b)} \bar{p}_{1}^{(b)}+\hat{C}^{(i, b)} \dot{\bar{p}}_{1}^{(b)} \\
& \dot{\bar{p}}_{2}^{(i)}=\dot{\bar{p}}_{q}^{(i)}+\dot{\hat{C}}^{(i, b)} \bar{p}_{2}^{(b)}+\hat{C}^{(i, b)} \dot{\bar{p}}_{2}^{(b)}
\end{aligned}
$$

Then, linear velocities of the links are given in quadrotor body-fixed reference frame in terms of respective Jacobimatrices, $\hat{J}$ further as: an

$$
\begin{aligned}
& \dot{\bar{p}}_{1}^{(b)}=\hat{J}_{v 1} \dot{\bar{\Omega}} \\
& \dot{\bar{p}}_{2}^{(b)}=\hat{J}_{v 2} \dot{\bar{\Omega}}
\end{aligned}
$$

Following that, Eq's. (7) and (8) can be further written as,

$$
\begin{aligned}
& \dot{\bar{p}}_{1}^{(i)}=\dot{\bar{p}}_{q}^{(i)}+\hat{C}^{(i, b)} \operatorname{SSM}\left(w_{q}^{(b)}\right) \bar{p}_{1}^{(b)}+\hat{C}^{(i, b)} \hat{J}_{v 1} \dot{\bar{\Omega}} \\
& \dot{\bar{p}}_{2}^{(i)}=\dot{\bar{p}}_{q}^{(i)}+\hat{C}^{(i, b)} \operatorname{SSM}\left(w_{q}^{(b)}\right) \bar{p}_{2}^{(b)}+\hat{C}^{(i, b)} \hat{J}_{v 2} \dot{\bar{\Omega}}
\end{aligned}
$$

Where in these equations SSM is for the skew symmetric matrix operation.

Angular velocity of the quadrotor with respect to the inertial frame can be written in body-fixed and inertial-fixed reference frames, $\bar{w}_{q}^{(b)}$ and $\bar{w}_{q}^{(i)}$, respectively as:

$$
\begin{aligned}
& \bar{w}_{q}^{(b)}=\hat{L} \dot{\bar{\Upsilon}} \\
& \bar{w}_{q}^{(i)}=\hat{C}^{(i, b)} \bar{w}_{q}^{(b)} \\
& \bar{w}_{q}^{(i)}=\hat{C}^{(i, b)} \hat{L} \dot{\bar{\Upsilon}}=\hat{T} \dot{\bar{\Upsilon}}
\end{aligned}
$$

Where,

$$
\hat{L}=\left[\begin{array}{ccc}
1 & 0 & -s \theta \\
0 & c \phi & c \theta s \phi \\
0 & -s \phi & c \theta c \phi
\end{array}\right]
$$

Where $\hat{L}$ is the matrix that maps the Euler angular rates to the angular velocities in the quadrotor body-fixed reference frame and maps the derivative of the Euler angles to the quadrotor's angular velocity in the inertial reference frame. Overhead dot (.) is used to represent the derivative of the corresponding variable.

The angular velocities of the links are given in the quadrotor body-fixed reference frame in terms of the Jacobian matrices as,

$$
\begin{aligned}
& \bar{w}_{1}^{(b)}=\hat{J}_{w 1} \dot{\bar{\Omega}} \\
& \bar{w}_{2}^{(b)}=\hat{J}_{w 2} \dot{\bar{\Omega}}
\end{aligned}
$$

Later, the angular velocities of the links in the inertialfixed reference frame are obtained as,

$$
\begin{aligned}
& \bar{w}_{1}^{(i)}=\bar{w}_{q}^{(i)}+\hat{C}^{(i, b)} \hat{J}_{w 1} \dot{\bar{\Omega}} \\
& \bar{w}_{2}^{(i)}=\bar{w}_{q}^{(i)}+\hat{C}^{(i, b)} \hat{J}_{w 2} \dot{\bar{\Omega}}
\end{aligned}
$$

Now, let us define the generalized coordinates, and the generalized velocities of the unified system as follows,

$$
\begin{aligned}
\bar{q} & =\left[\begin{array}{llllllll}
x & y & z & \phi & \theta & \psi & \theta_{1} & \theta_{2}
\end{array}\right]^{T} \\
\dot{\bar{q}} & =\left[\begin{array}{llllllll}
\dot{x} & \dot{y} & \dot{z} & \dot{\phi} & \dot{\theta} & \dot{\psi} & \dot{\theta}_{1} & \dot{\theta}_{2}
\end{array}\right]^{T}
\end{aligned}
$$

By using the generalized velocities, the linear and angular velocities with respect to the inertial-fixed reference frame can be written further in terms of velocity influence coefficients as [18].

$$
\begin{aligned}
& \dot{\bar{p}}_{q}^{(i)}=\left[\begin{array}{lll}
\hat{I}_{3 \times 3} & \hat{0}_{3 x 5}
\end{array}\right] \dot{\bar{q}}=\hat{V}_{q}^{\dot{\bar{q}}} \\
& \bar{w}_{q}^{(i)}=\left[\begin{array}{lll}
\hat{0}_{3 \times 3} & \hat{T} & \hat{0}_{3 \times 2}
\end{array}\right] \dot{\bar{q}}=\hat{W}_{q} \dot{\bar{q}} \\
& \dot{\bar{p}}_{1}^{(i)}=\left[\begin{array}{lll}
\hat{I}_{3 \times 3} & -\hat{C}^{(i, b)} \operatorname{SSM}\left(\bar{p}_{1}^{(b)}\right) \hat{L} & \hat{C}^{(i, b)} \hat{J}_{v 1}
\end{array}\right] \dot{\bar{q}}=\hat{V}_{1} \dot{\bar{q}} \\
& \dot{\bar{p}}_{2}^{(i)}=\left[\begin{array}{lll}
\hat{I}_{3 \times 3} & -\hat{C}^{(i, b)} \operatorname{SSM}\left(\bar{p}_{2}^{(b)}\right) \hat{L} & \hat{C}^{(i, b)} \hat{J}_{v 2}
\end{array}\right] \dot{\bar{q}}=\hat{V}_{2} \dot{\bar{q}} \\
& \bar{w}_{1}^{(i)}=\left[\begin{array}{lll}
\hat{0}_{3 \times 3} & \hat{T} & \hat{C}^{(i, b)} \hat{J}_{w 1}
\end{array}\right] \dot{\bar{q}}=\hat{W}_{1} \dot{\bar{q}} \\
& \bar{w}_{2}^{(i)}=\left[\begin{array}{lll}
\hat{0}_{3 \times 3} & \hat{T} & \hat{C}^{(i, b)} \hat{J}_{w 2}
\end{array}\right] \dot{\bar{q}}=\hat{W}_{2} \dot{\bar{q}}
\end{aligned}
$$

In above equations, $\hat{V}$ and $\hat{W}$ are the linear and angular velocity influence coefficients, respectively.

\section{Dynamics}

Dynamic model of the unified quadrotor and the robotic arm is obtained by using Lagrange-d'Alembert formulation [17]. It can be written as,

$$
\begin{aligned}
& \frac{d}{d t}\left(\frac{\partial L}{\partial \dot{\bar{q}}}\right)-\frac{\partial L}{\partial \bar{q}}=\bar{u}+\bar{u}_{e x t} \\
& L=K-U
\end{aligned}
$$

where $L$ is the Lagrange operator, $K$ and $U$ are the total kinetic and potential energies of the combined system. The terms $\bar{u}$ and $\bar{u}_{\text {ext }}$ are the generalized control input and the external interaction forces that are applied to end-effector, respectively. 
The total kinetic energy, $K$ is written as the summation of the kinetic energies of the each mass elements that are quadrotor $\operatorname{mass}\left(m_{b}\right)$, link-1 $\operatorname{mass}\left(m_{1}\right)$ and link-2 $\operatorname{mass}\left(m_{2}\right)$.

$$
\begin{aligned}
& K=K_{b}+K_{1}+K_{2} \\
& K_{b}=\frac{1}{2} \dot{\bar{p}}_{q}^{(i) T} m_{b} \dot{\bar{p}}_{q}^{(i)}+\frac{1}{2} \bar{\omega}_{q}^{(i) T} \hat{C}^{(i, b)} \hat{I}_{b} \hat{C}^{(i, b) T} \bar{\omega}_{q}^{(i)} \\
& K_{1}=\frac{1}{2} \dot{\bar{p}}_{1}^{(i) T} m_{1} \dot{\bar{p}}_{1}^{(i)}+\frac{1}{2} \bar{\omega}_{1}^{(i) T} \hat{C}^{(i, b)} \hat{C}^{(b, 1)} \hat{I}_{1} \hat{C}^{(b, 1) T} \hat{C}^{(i, b) T} \bar{\omega}_{1}^{(i)} \\
& K_{2}=\frac{1}{2} \dot{\bar{p}}_{2}^{(i) T} m_{2} \dot{\bar{p}}_{2}^{(i)}+\frac{1}{2} \bar{\omega}_{2}^{(i) T} \hat{C}^{(i, b)} \hat{C}^{(b, 2)} \hat{I}_{2} \hat{C}^{(b, 2) T} \hat{C}^{(i, b) T} \bar{\omega}^{(i)}
\end{aligned}
$$

In these equations, $\hat{I}$ is the constant inertia matrix at the corresponding reference frames of the vehicle body and the links bodies.

The total potential energy of the unified system can be expressed as,

$$
\begin{aligned}
& U=U_{b}+U_{1}+U_{2} \\
& U_{b}=m_{b} g \bar{u}_{3}^{t} \bar{p}_{q}^{(i)} \\
& U_{1}=m_{1} g \bar{u}_{3}^{t} \bar{p}_{1}^{(i)} \\
& U_{2}=m_{2} g \bar{u}_{3}^{t} \bar{p}_{2}^{(i)}
\end{aligned}
$$

where $g$ is the gravity. After the Eq's. (29) and (33) are plugged into Eq. (28) the following form of the equation of motion of the unified system can be obtained as:

$$
\hat{M}(\bar{q}) \ddot{\bar{q}}+\hat{C}(\bar{q}, \dot{\bar{q}}) \dot{\bar{q}}+\hat{G}(\bar{q})=\bar{u}+\bar{u}_{e x t}
$$

where $\hat{M}$ is positive definite and symmetric inertia matrix, $\hat{C}$ consists of centripetal, Coriolis, gyroscopic terms, and $\hat{G}$ consists of gravity terms.

Alternatively, to find these matrices, the following formulations are used. The total kinetic energy can be expressed in terms of the inertia matrix as [18],

$$
K=\frac{1}{2} \dot{\bar{q}}^{T} \hat{M}(\bar{q}) \dot{\bar{q}}
$$

By using the Eq's. (22), (23), (24), (25), (26), (27), and (38), the inertia matrix can be further written as,

$$
\begin{aligned}
& \hat{M}(\bar{q})=\hat{V}_{q}^{T} m_{b} \hat{V}_{q}+\hat{W}_{q}^{T} \hat{C}^{(i, b)} \hat{I}_{b} \hat{C}^{(i, b) T} \hat{W}_{q}+ \\
& \sum_{k=1}^{2} \hat{V}_{k}^{T} m_{k} \hat{V}_{k}+\hat{W}_{k}^{T}\left(\hat{C}^{(i, b)} \hat{C}^{(b, k)}\right) \hat{I}_{k}\left(\hat{C}^{(i, b)} \hat{C}^{(b, k)}\right)^{T} \hat{W}_{k}
\end{aligned}
$$

Also, the elements of the Coriolis matrix can be expressed as follows [19].

$$
c_{a, b}=\sum_{j=1}^{8} \frac{1}{2}\left\{\frac{\partial m_{a, b}}{\partial q_{j}}+\frac{\partial m_{a, j}}{\partial q_{b}}-\frac{\partial m_{j, b}}{\partial q_{a}}\right\}
$$

Finally, the column matrix $\hat{G}$ can be obtained by using the total potential energy as,

$$
\hat{G}(\bar{q})=\frac{\partial U}{\partial \bar{q}}
$$

The generalized input force, $\bar{u}$ and the externally applied disturbance forces, $\bar{u}_{\text {ext }}$ are obtained by using the virtual work principle method and expressed as follows.

$$
\bar{u}=\hat{S}\left[\begin{array}{c}
\bar{f}_{q}^{(b)} \\
\bar{\tau}_{q}^{(b)} \\
\bar{\tau}_{12}
\end{array}\right]=\left[\begin{array}{ccc}
\hat{C}^{(i, b)} & \hat{0}_{3 \times 3} & \hat{0}_{3 x 2} \\
\hat{0}_{3 \times 3} & \hat{L}^{t} & \hat{0}_{3 \times 2} \\
\hat{0}_{2 \times 3} & \hat{0}_{2 \times 3} & \hat{I}_{2 \times 2}
\end{array}\right]\left[\begin{array}{c}
\bar{f}_{q}^{(b)} \\
\bar{\tau}_{q}^{(b)} \\
\bar{\tau}_{12}
\end{array}\right]
$$

where, $\operatorname{det}(\hat{S})=\cos (\theta)$

Therefore, if $\theta \neq \pi n-\frac{\pi}{2}, n \in Z$ then, $\hat{S}$ is an invertible matrix. For our case, the pitch angle satisfies this condition.

$$
\bar{u}_{e x t}=\left[\begin{array}{cc}
\hat{I}_{3 x 3} & \hat{0}_{3 x 3} \\
\operatorname{SSM}\left(\bar{p}_{e}^{(b)}\right) & \hat{I}_{3 x 3} \\
\hat{J}_{e v}^{T} & \hat{J}_{e \omega}^{T}
\end{array}\right] \bar{P}
$$

where the subscripts ' ${ }^{\prime *}$ "' shows the size of the matrices. $\bar{p}_{e}^{(b)}$ is the position of the end effector in quadrotor body fixed frame. $\bar{f}_{q}^{(b)}$ and $\bar{\tau}_{q}^{(b)}$ are the forces and torques generated by the quadrotor motors, and $\bar{\tau}_{12}$ is the vector of arm's joint torques.

$$
\bar{f}_{q}^{(b)}=\left[\begin{array}{lll}
0 & 0 & f_{z}
\end{array}\right]^{T}, \quad \bar{\tau}_{q}^{(b)}=\left[\begin{array}{lll}
\tau_{q 1} & \tau_{q 2} & \tau_{q 3}
\end{array}\right]^{T}
$$

Also, $\bar{P}$ is the vector of the interaction force, $\bar{F}$ and moment, $\hat{M}$.

$$
\bar{P}=\left[\begin{array}{llllll}
F_{1} & F_{2} & F_{3} & M_{1} & M_{2} & M_{3}
\end{array}\right]^{T}
$$

In addition, the quadrotor's rotors rotational speeds, $\varpi$ 's can be mapped to the generated forces and torques by the following relation.

$$
\left[\begin{array}{c}
f_{z} \\
\tau_{q 1} \\
\tau_{q 2} \\
\tau_{q 3}
\end{array}\right]=\left[\begin{array}{cccc}
-c_{T} & -c_{T} & -c_{T} & -c_{T} \\
0 & -d c_{T} & 0 & d c_{T} \\
d c_{T} & 0 & -d c_{T} & 0 \\
-c_{Q} & c_{Q} & -c_{Q} & c_{Q}
\end{array}\right]\left[\begin{array}{l}
\varpi_{1}^{2} \\
\varpi_{2}^{2} \\
\varpi_{3}^{2} \\
\varpi_{4}^{2}
\end{array}\right]
$$

Where $c_{T}$ and $c_{Q}$ are the dc motors' thrust and drag coefficients, respectively. $\mathrm{d}$ is the distance between one $\mathrm{dc}$ motor and the center of mass of the quadrotor. The above relation is obtained by assuming that the square of the rotational speeds of each rotor is proportional to the generated forces and moments [1].

\section{CONTROLLER DESIGN}

The decoupled controllers are designed for the position and attitude control of the quadrotor and the angular po- 
sition control of the robotic arm joints. While developing controller algorithms, the linearized decoupled equations of motion of the combined system is used. The controller architecture of the combined system is depicted in Fig. 2. The controller contains an outer and an inner loop.

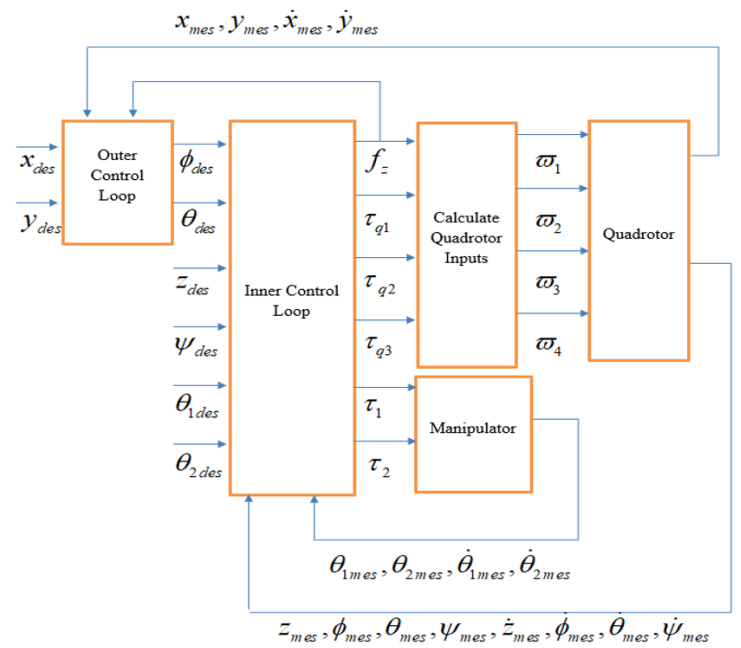

Figure 2. The controller architecture of the unified system

\section{Outer Loop Control}

Quadrotor is an underactuated vehicle that has four control inputs while having 6-DOF to be controlled. In other words, in order to have a translation motion in $\mathrm{x}$-direction, the pitch angle should be changed. The same logic is valid for the translational motion in $y$-direction. The difference is that the roll angle should be changed. Therefore, these four states are heavily coupled with each other. To overcome this problem, an outer loop controller designed to compute the motion references for the roll and pitch angles from the reference values of the translation in $\mathrm{x}$ and $\mathrm{y}$ directions.

\section{$x$ and $y$ Position Controllers}

In this controller, for the desired positions in $\mathrm{x}$ and $\mathrm{y}$, intermediate control inputs $\theta$ and $\phi$ are calculated and fed to the inner loop controller. To obtain control law, the following relation is written by using Eq. (42).

$$
\begin{aligned}
& u_{1}=f_{z}(\cos (\psi) \sin (\theta) \cos (\phi)+\sin (\psi) \sin (\phi)) \\
& u_{2}=f_{z}(\sin (\psi) \sin (\theta) \cos (\phi)-\cos (\psi) \sin (\phi))
\end{aligned}
$$

By putting $\psi=0$, Eq. (47) can be written further as for the desired roll and pitch angles as,

$$
\begin{aligned}
& u_{1}=m \ddot{x}=f_{z} \theta \\
& u_{2}=m \ddot{y}=-f_{z} \phi
\end{aligned}
$$

For Eq. (48), following control law is obtained via PID control law with feedback linearizing logic. Where e is the error between desired (des) and measured (mes) states.

$$
\begin{gathered}
e_{x}=x_{d e s}-x_{m e s} \\
e_{y}=y_{d e s}-y_{m e s} \\
\theta_{d e s}=\frac{m}{f_{z}}\left(\ddot{x}_{d e s}+K_{p x} e_{x}(t)+K_{i x} \int_{0}^{t} e_{x}(\tau) d \tau+K_{d x} \frac{d e_{x}(t)}{d t}\right) \\
\phi_{d e s}=-\frac{m}{f_{z}}\left(\ddot{y}_{d e s}+K_{p y} e_{y}(t)+K_{i y} \int_{0}^{t} e_{y}(\tau) d \tau+K_{d y} \frac{d e_{y}(t)}{d t}\right)
\end{gathered}
$$

Where $K_{p}, K_{i}$, and $K_{d}$ are the proportional, integral and the derivative gains of the PID controller. $m$ is the total mass of the combined system.

\section{Inner Loop Control}

The calculated roll and pitch angles are used as reference values for the roll and pitch controllers. For yaw and altitude controllers, reference values are fed from the outside.

\section{Altitude Controller}

A PID control law is proposed by using the following relation that is obtained from Eq. (42).

$$
u_{3}=m \ddot{z}=f_{z}+m g
$$

The control input can be calculated by using following relation.

$$
f_{z}=m\left(\ddot{z}_{d e s}+K_{p z} e_{z}(t)+K_{i z} \int_{0}^{t} e_{z}(\tau) d \tau+K_{d z} \frac{d e_{z}(t)}{d t}\right)-m g
$$

\section{Attitude Controller}

From Eq. (42), the following relations can be written.

$$
\begin{aligned}
& u_{4}=I_{x x} \ddot{\phi}=\tau_{q 1} \\
& u_{5}=I_{y y} \ddot{\theta}=\tau_{q 2} \\
& u_{6}=I_{z z} \ddot{\psi}=\tau_{q 3}
\end{aligned}
$$

The attitude controllers have a cascaded structure as in Fig. 3. There is an inner loop that regulates the angular velocity and there is an outer loop that controls the angular position. Inner loop is stabilized by the PI controller and the outer loop is controlled by the P controller. The control inputs can be written as follows,

$$
\begin{aligned}
& e_{\dot{\phi}}=\dot{\phi}_{d e s}-\dot{\phi}_{m e s} \\
& \tau_{q 1}=\left(K_{p 2 \phi} e_{\phi}(t)-\dot{\phi}\right)\left[K_{p \dot{\phi}} e_{\dot{\phi}}(t)+K_{i \dot{\phi}} \int_{0}^{t} e_{\dot{\phi}}(\tau) d \tau\right] \\
& \tau_{q 2}=\left(K_{p 2 \theta} e_{\theta}(t)-\dot{\theta}\right)\left[K_{p \dot{\theta}} e_{\dot{\theta}}(t)+K_{i \dot{\theta}} \int_{0}^{t} e_{\dot{\theta}}(\tau) d \tau\right] \\
& \tau_{q 3}=\left(K_{p 2 \psi} e_{\psi}(t)-\dot{\psi}\right)\left[K_{p \dot{\psi}} e_{\dot{\psi}}(t)+K_{i \dot{\psi}} \int_{0}^{t} e_{\dot{\psi}}(\tau) d \tau\right]
\end{aligned}
$$




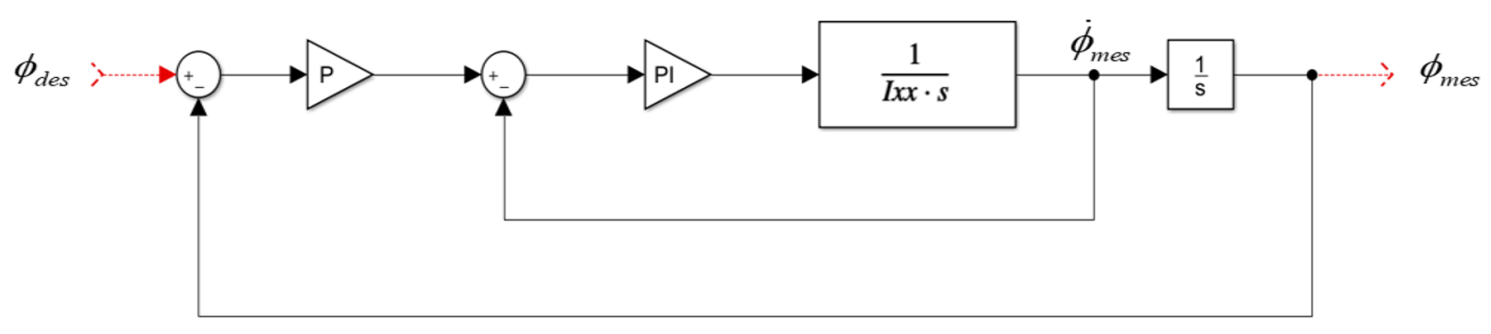

Figure 3. Roll Controller Structure

\section{Robotic Arm Joint Angles Controller}

For the joints, the following relations are used to obtain PID control laws. In these equations, $I_{12}$ is total the inertia of the two arms about their total mass centers and $I_{2}$ is the inertia of the second link. With the small joint angle assumptions, the following relations are a good approximation for the controller design.

$$
\begin{aligned}
& I_{12} \ddot{\theta}_{1}=\tau_{1}-m_{12} g \sin \left(\theta_{1}\right)\left(\frac{b_{1}+b_{2}}{2}\right) \\
& I_{2} \ddot{\theta}_{2}=\tau_{2}-m_{2} g \sin \left(\theta_{2}\right)\left(\frac{b_{2}}{2}\right)
\end{aligned}
$$

From the above equations, by using feedback linearizing logic, the control inputs can be written as,

$$
\begin{aligned}
& \tau_{1}=I_{12}\left(\ddot{\theta}_{1 d e s}+\tau_{\theta 1}\right)+m_{12} g \sin \left(\theta_{1}\right)\left(\frac{b_{1}+b_{2}}{2}\right) \\
& \tau_{\theta 1}=K_{p \theta 1} e_{\theta 1}(t)+K_{i \theta 1} \int_{0}^{t} e_{\theta 1}(\tau) d \tau+K_{d \theta 1} \frac{d e_{\theta 1}(t)}{d t} \\
& \tau_{2}=I_{2}\left(\ddot{\theta}_{2 d e s}+\tau_{\theta 2}\right)+m_{2} g \sin \left(\theta_{2}\right)\left(\frac{b_{2}}{2}\right) \\
& \tau_{\theta 2}=K_{p \theta 2} e_{\theta 2}(t)+K_{i \theta 2} \int_{0}^{t} e_{\theta 2}(\tau) d \tau+K_{d \theta 2} \frac{d e_{\theta 2}(t)}{d t}
\end{aligned}
$$

\section{SIMULATION RESULTS}

The performance of the controllers are assessed using simulation-based analysis as detailed in this section.

\section{Simulation Parameters and Scenario}

The proposed control algorithms are tested in MATLAB/ Simulink simulation environment. For the controller outputs, the saturation blocks are added to avoid unrealistic inputs to the system. The rotational speeds of the rotors are limited to the $800 \mathrm{rad} / \mathrm{sec}$, the torque inputs of the servo motors are limited to the $3 \mathrm{Nm}$ and the intermediate control inputs; phi and theta are limited to the $17 \mathrm{deg}$ -

\begin{tabular}{|c|c|c|c|c|c|c|c|c|}
\hline & $x$ & $y$ & $z$ & $\phi$ & $\theta$ & $\psi$ & $\theta_{1}$ & $\theta_{2}$ \\
\hline$K_{p}$ & 24.790 & 24.790 & 39.430 & 1.959 & 1.959 & 1.269 & 1325 & 4669 \\
\hline$K_{i}$ & 12.360 & 12.360 & 27.880 & 20.880 & 20.880 & $4 \cdot 390$ & 6169 & 40370 \\
\hline$K_{d}$ & $9 \cdot 407$ & 9.407 & 12.170 & - & - & - & 70.450 & 129.800 \\
\hline$K_{p 2}$ & - & - & - & 9.172 & 9.172 & 3.339 & - & - \\
\hline
\end{tabular}
rees. The numerical parameters of the combined system are presented in the Table 2.
Table 2. Numerical Parameters of the Combined System

\begin{tabular}{cccc}
\hline$m_{b}[\mathrm{~kg}]$ & 2.6550 & $d[\mathrm{~m}]$ & 0.3435 \\
\hline$m_{1,2}[\mathrm{~kg}]$ & 0.1700 & $b_{0}[\mathrm{~m}]$ & 0.3435 \\
$l_{x x, b}\left[\mathrm{kgm}^{2}\right]$ & 0.0457 & $b_{1,2}[\mathrm{~m}]$ & 0.3000 \\
$l_{y y, b}\left[\mathrm{kgm}^{2}\right]$ & 0.0457 & $c_{1,2}[\mathrm{~m}]$ & 0.0500 \\
$l_{z z, b}\left[\mathrm{kgm}^{2}\right]$ & 0.0846 & $d_{1,2}[\mathrm{~m}]$ & 0.0500 \\
$l_{x x, 12}\left[\mathrm{kgm}^{2}\right]$ & $7.0830 e-05$ & $g r a v i t y\left[\mathrm{~m} / \mathrm{s}^{2}\right]$ & 9.8100 \\
$l_{y y, 12}\left[\mathrm{kgm}^{2}\right]$ & 0.0013 & $c_{T}\left[\mathrm{~N} /(\mathrm{rad} / \mathrm{s})^{2}\right]$ & $2.7400 \mathrm{e}-05$ \\
$l_{z z, 12}\left[\mathrm{kgm}^{2}\right]$ & 0.0013 & $c_{0}\left[\mathrm{Nm} /(\mathrm{rad} / \mathrm{s})^{2}\right]$ & $0.0470 \mathrm{e}-05$ \\
\hline
\end{tabular}

Table 3. The Gains of the Proposed Controllers

Table 4. The Reference Values of the Controlled Variables

\begin{tabular}{ccccc}
\hline Time $[\mathrm{s}]$ & $0-9$ & $10-19$ & $25-30$ & $30-60$ \\
\hline$x[\mathrm{~m}]$ & $0-5$ & 5 & 5 & 5 \\
$y[\mathrm{~m}]$ & $0-3$ & 3 & 3 & 3 \\
$z[\mathrm{~m}]$ & - & -2 & -2 & -2 \\
$\phi[\mathrm{deg}]$ & - & - & - & - \\
$\theta[\mathrm{deg}]$ & $0-(-5)$ & - & - & - \\
$\psi[\mathrm{deg}]$ & 0 & -5 & -5 & -5 \\
$\theta_{1}[\mathrm{deg}]$ & 0 & $0-15$ & 15 & 15 \\
$\theta_{2}[\mathrm{deg}]$ & 0 & $0-10$ & 10 & 10 \\
$F_{1}[\mathrm{~N}]$ & 0 & 0 & $0-5$ & 5 \\
$F_{2}[\mathrm{~N}]$ & 0 & 0 & $0-2$ & 2 \\
$F_{3}[\mathrm{~N}]$ & 0 & 0 & $0-6$ & 6 \\
\hline
\end{tabular}




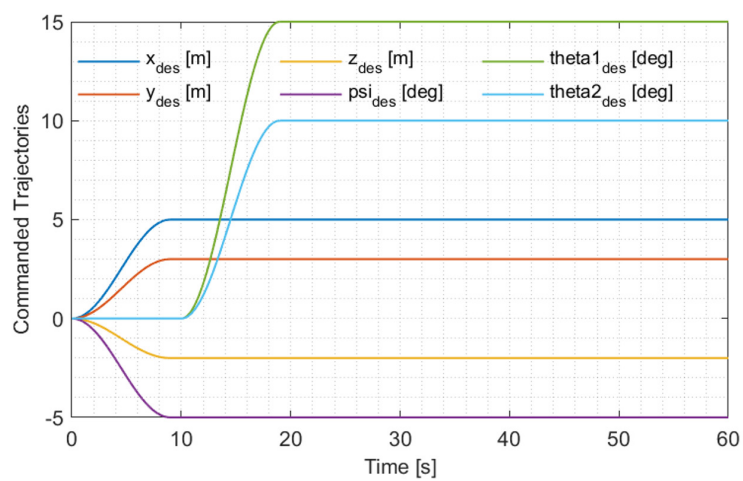

Figure 4. The Desired Values of the Directly Controlled Parameters

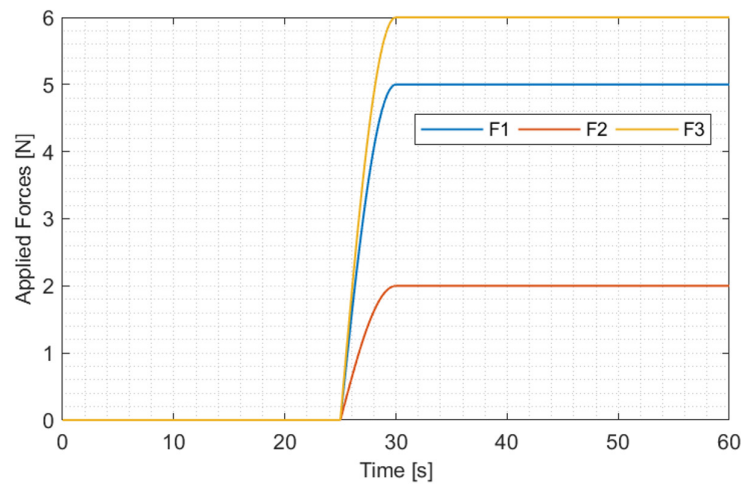

Figure 5. The Applied Disturbance Forces

The gains of the designed controllers are given in Table 3. These gains are obtained by using the root locus method of the classical control theory.

The proposed controllers are tested with a simulation scenario. This scenario is shown in Table 4 and Fig. 4.

Firstly, the quadrotor is commanded to move to the predefined positions ( $5 \mathrm{~m}$ in $\mathrm{x}, 3 \mathrm{~m}$ in $\mathrm{y}$, and $-2 \mathrm{~m}$ in $\mathrm{z}$ ) and yaw angle joint angles of the robotic arm are kept at 0 . Then, the robotic arm is moved to the predefined angles ( 15 degree in $\theta_{1}$ and 10 degree in $\theta_{2}$ ). Finally, the disturbance forces are applied to the end-effector of the serial manipulator $(6 \mathrm{~N}$ in $+\mathrm{x}$-direction, $2 \mathrm{~N}$ in $+\mathrm{y}$-direction and $6 \mathrm{~N}$ in $+\mathrm{z}$-direction).

\section{Quadrotor Position Control}

The performance of the position controller is evaluated and the response of the aerial manipulator in the inertial reference frame is depicted in Fig. 6 and Fig. 7.

Although the robotic arm is active and external disturbances are applied to the system, the quadrotor tracked the input well (Fig. 6) and the maximum absolute error is less than 0.03 m during the course of the motion (Fig. 7).

\section{Quadrotor Attitude Control}

Due to the underactuated nature of the quadrotors, the
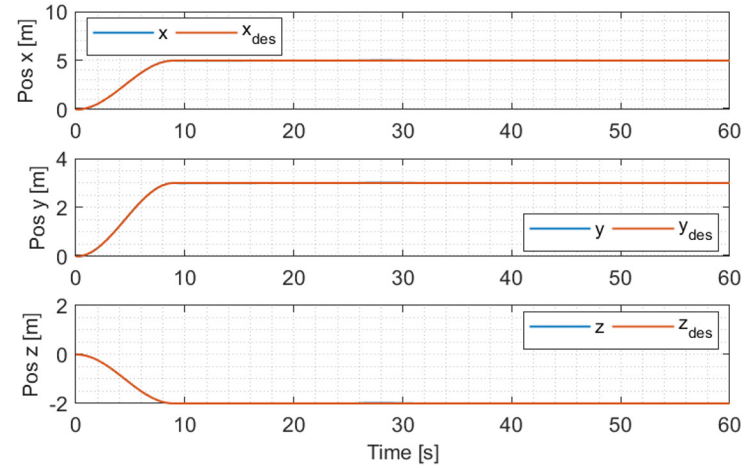

Figure 6. The Desired and Achieved Cartesian Position of the Quadrotor

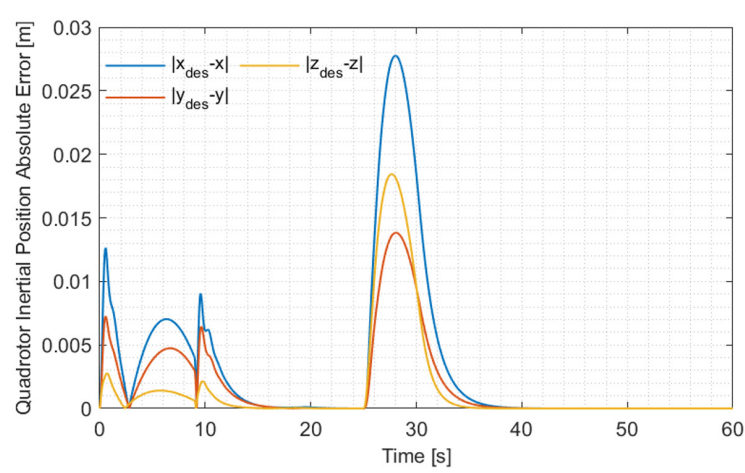

Figure 7. The Absolute Error Between Desired and Achieved Inertial Position of the Quadrotor

attitude dynamics of quadrotors is manipulated to move the quadrotor to a desired position. Fig. 8 and Fig. 9 present the reference and actual values of the Euler angles during the position control in Section 4.2. Fig. 10 shows the corresponding absolute error due to the difference between the desired and achieved values.

Due to the underactuated nature of the system, the performance of the attitude control system is effective in the performance of position control. The quadrotor tracks the desired attitude values well (Fig. 8). During the transient period, the maximum error is around -4 degrees in theta and 2 degrees in phi (Fig. 9). The maximum absolute error is almost 1.2 degrees in theta (Fig. 10).
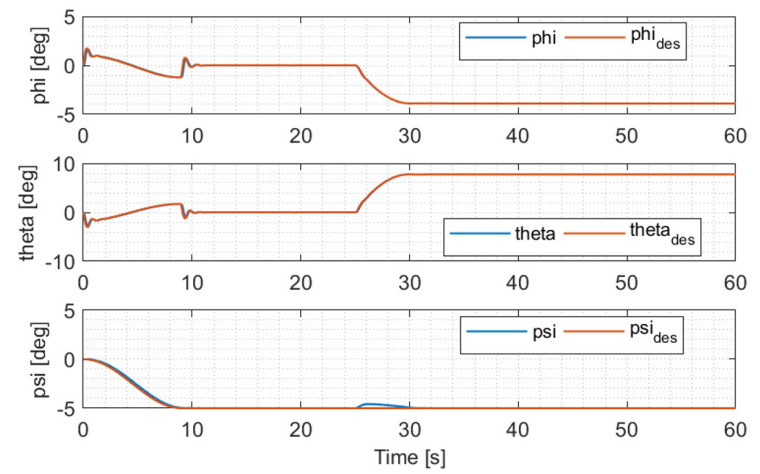

Figure 8. The Desired and Achieved Attitude of the Quadrotor 

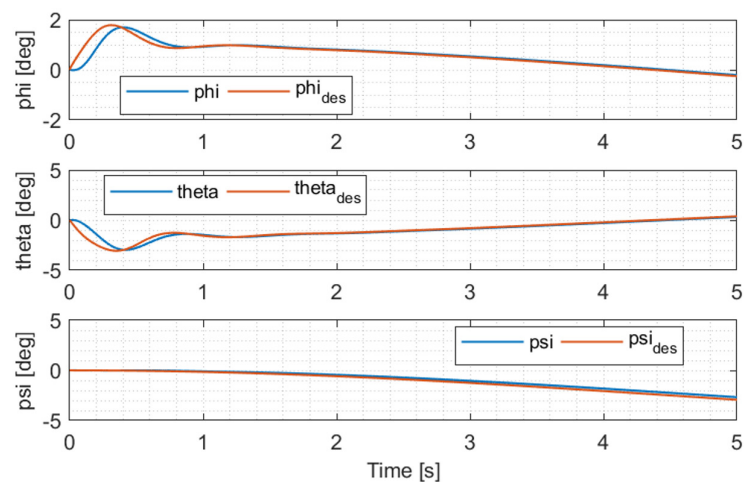

Figure 9. The Zoomed Version of the Desired and Achieved Attitude of the Quadrotor

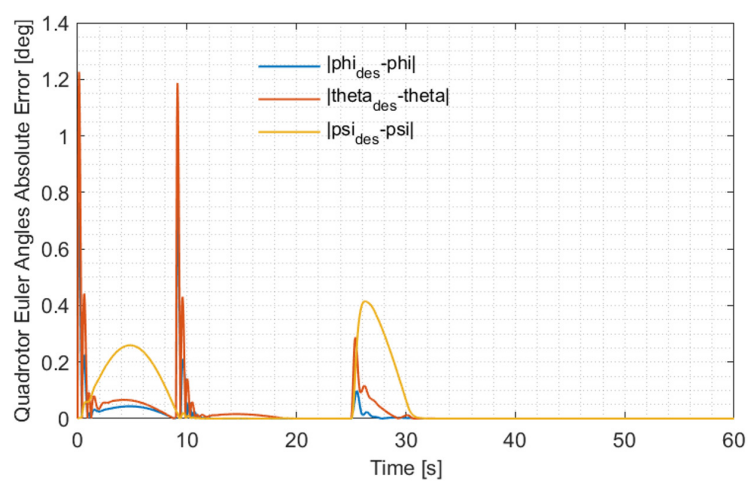

Figure 10. The Absolute Error Between the Desired and Achieved Attitude of the Quadrotor

\section{Robotic Arm Joint Angles Control}

The coupled arm is controlled during the position control experiment. The reference and actual values of the joint angles is depicted in Fig. 11 and its zoomed version is depicted in Fig. 12. Fig. 13 shows the corresponding absolute error due to the difference in the reference and the actual values.

The robotic arm tracked the desired angles well both in the steady state (Fig. 11) and transient (Fig. 12) periods. The maximum absolute error in both axes is less than 0.5 degrees (Fig. 13).
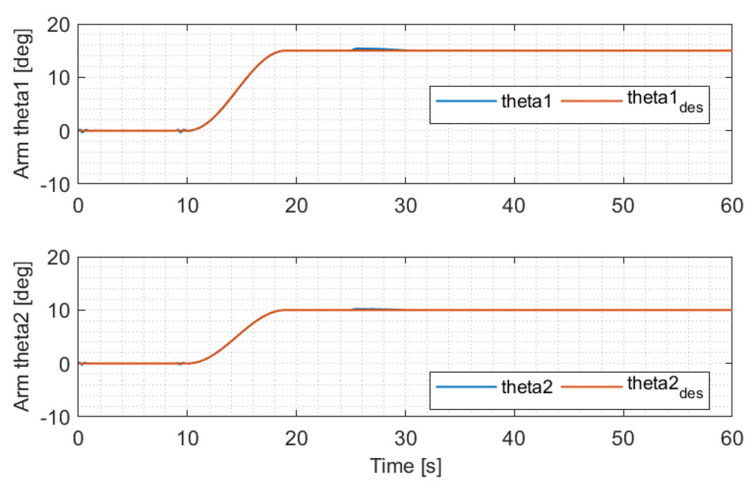

Figure 11. The Desired and Achieved Joint Angles of the Robotic Arm
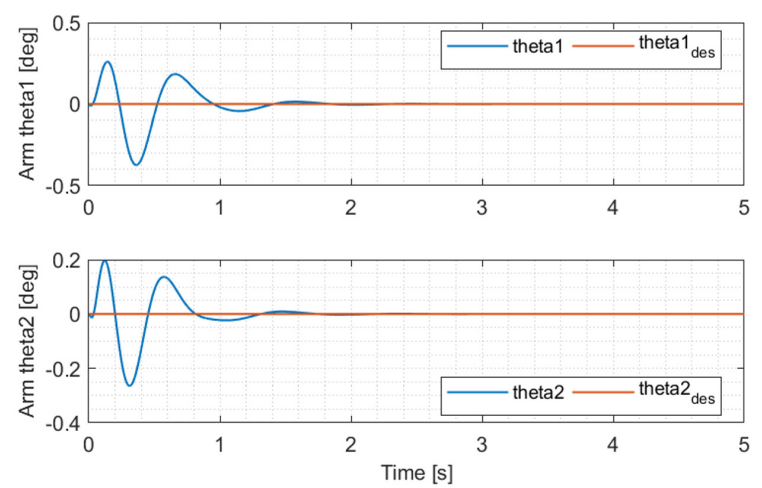

Figure 12. The Zoomed Version of the Desired and Achieved Joint Angles of the Robotic Arm

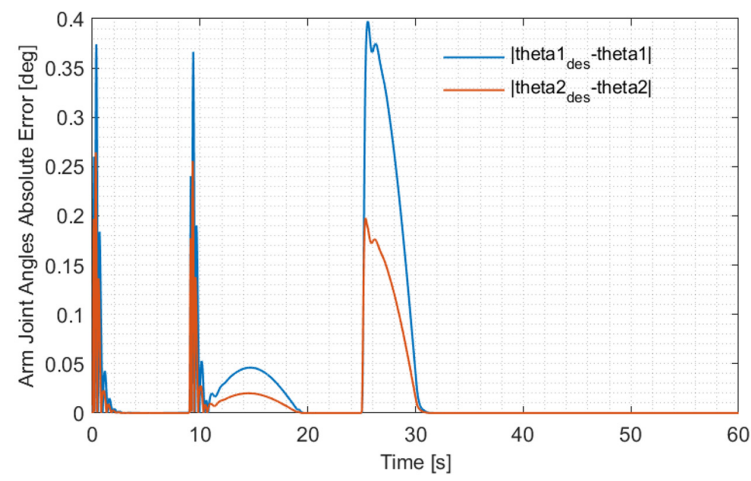

Figure 13. The Absolute Error Between the Desired and Achieved Joint Angles of the Robotic Arm

\section{DISCUSSION AND CONCLUSION}

In this paper, decoupled controllers are proposed for an aerial manipulation system composed of a quadrotor and a serial 2-DOF robotic arm. First, for the position of the quadrotor and joint angles, decoupled PID controllers are designed by using feedback linearization technique. Then, cascaded PID controllers are proposed for the attitude dynamics of the quadrotor. Here, reference values of the roll and pitch angle of the quadrotor are the output of the position controllers of the $\mathrm{x}$ and $\mathrm{y}$. Designed controllers are tested in a simulation environment with highly nonlinear dynamic models.

At first, nonzero cartesian positions and yaw angle are commanded to the quadrotor. The aerial manipulator reaches these reference trajectories in 9 seconds. Due to the transient and coupled dynamics of the system, there are some oscillations. However, these oscillations are suppressed with the action of the controllers (Figs. 6, 7 and 8). Also, there are some small deviations from the zero reference values of the joint angles as shown in Figs. 11, 12 and 13. This is caused by the faster pitch dynamics of the quadrotor and the transient dynamics of the system. These oscillations are controlled by the joint controllers. Then, at 10 seconds, the serial manipulator is commanded to track the reference values. The joint controllers track these references successfully. 
The quadrotor is affected from this action slightly, but it is suppressed by position controllers. Finally, the disturbance forces are applied to the end-effector of the serial manipulator at 25 seconds as shown in Fig. 5. The quadrotor deviates from its original position, but it comes to the reference position very fast with the action of the controllers. The attitude controllers tilt the quadrotor to balance these multi-directional forces as illustrated. Thus, it has nonzero roll and pitch angles as illustrated in Fig. 9 after the disturbance forces are applied. The joint controllers deal with these disturbances very effectively.

To sum up, the controllers' performances are tested with the coupled reference commands and disturbance forces. The performance of the controllers is found to be satisfactory.

As a future work, the sensor models of the accelerometer, gyro, and the indoor localization system will be implemented into simulation environment. Then, the gains of the controllers will be updated. Finally, we plan to implement the developed controller algorithms in a real system.

\section{References}

1. Kotarski, Denis, Zoran Beni, and Matija Krznar. Control design for unmanned aerial vehicles with four rotors. Interdisciplinary Description of Complex Systems: INDECS 14.2 (2016) 236-245.

2. Das, Abhijit, Kamesh Subbarao, and Frank Lewis. Dynamic inversion with zero-dynamics stabilisation for quadrotor control. IET Control Theory and Applications 3.3 (2009) 303-314.

3. Sadr, Sara, S. Ali A. Moosavian, and Payam Zarafshan. Dynamics modeling and control of a quadrotor with swing load. Journal of Robotics 2014 (2014) 1-12.

4. Mahony, Robert, Vijay Kumar, and Peter Corke. Multirotor aerial vehicles: Modeling, estimation, and control of quadrotor. IEEE Robotics and Automation Magazine 19.3 (2012) 20-32.

5. Goodarzi FA, Lee D, Lee T. Geometric stabilization of a quadrotor UAV with a payload connected by flexible cable. In 2014 American Control Conference, pp. 4925-4930, 2014.

6. Sreenath K, Kumar V. Dynamics, control and planning for cooperative manipulation of payloads suspended by cables from multiple quadrotor robots. In Robotics: Science and Systems IX, 2013.

7. Alothman, Y., Guo, M., \& Gu, D. Using iterative LQR to control two quadrotors transporting a cable-suspended load. IFACPapersOnLine 50.1 (2017) 4324-4329.

8. Kim S, Choi S, Kim HJ. Aerial manipulation using a quadrotor with a two DOF robotic arm. In 2013 IEEE/RSJ International Conference on Intelligent Robots and Systems, pp. 49904995. 2013.

9. Caccavale, F., Giglio, G., Muscio, G., \& Pierri, F. Adaptive control for UAVs equipped with a robotic arm. IFAC Proceedings Volumes 47.3 (2014) 11049-11054.

10. Jimenez-Cano A, Martin J, Heredia G, Ollero A, Cano R. Control of an aerial robot with multi-link arm for assembly tasks. In 2013 IEEE International Conference on Robotics and Automation, pp. 4916-4921, 2013.

11. Danko TW, Chaney KP, Oh PY. A parallel manipulator for mobile manipulating UAVs. In 2015 IEEE International Conference on Technologies for Practical Robot Applications (TePRA), pp. 1-6, 2015.

12. Orsag M, Korpela C, Bogdan S, Oh P. Lyapunov based model reference adaptive control for aerial manipulation. In 2013 International Conference on Unmanned Aircraft Systems (ICUAS), pp. 966-973, 2013.

13. Khalifa A, Fanni M. A new quadrotor manipulation system: Modeling and point-to-point task space control. International Journal of Control, Automation and Systems 15.3 (2017) 1434-46.

14. Giglio G, Pierri F. Selective compliance control for an unmanned aerial vehicle with a robotic arm. In 22nd Mediterranean Conference on Control and Automation, pp. 1190-1195, 2014.

15. Mello LS, Raffo GV, Adorno BV. Robust whole-body contro of an unmanned aerial manipulator. In 2016 European Control Conference (ECC), pp. 702-707, 2016.

16. Garimella G, Kobilarov M. Towards model-predictive control for aerial pick-and-place. In 2015 IEEE International Conference on Robotics and Automation (ICRA), pp. 46924697, 2015.

17. Bulut, N., Turgut, A. E., \& Arıkan, K. B. (2019). Computed Torque Control of an Aerial Manipulation System with a Quadrotor and a 2-DOF Robotic Arm. In 2019 International Conference on Informatics in Control, Automation and Robotics (ICINCO), 2019.

18. Ozgoren, M. K. Lecture Notes for ME 522 (Principles of Robotics), Department of Mechanical Engineering, METU, Ankara, 2017.

19. Siciliano B, Sciavicco L, Villani L, Oriolo G. Robotics: Modelling, planning and control, Springer, London, 2010. 